\title{
Influence of boundary conditions on strain field analysis for polycrystalline finite element simulations
}

\author{
Eva Héripré* - Jérôme Crépin* — Arjen Roos** \\ Jean-Louis Chaboche** \\ * Solid Mechanics Laboratory, CNRS UMR 7649 \\ Department of Mechanics, Ecole Polytechnique, F-91128 Palaiseau cedex \\ \{heripre,crepin\}@lms.polytechnique.fr \\ ** ONERA DMSM/CEMN \\ 29 avenue de la Division Leclerc, BP 72, F-92322 Châtillon cedex \\ \{arjen.roos, jean-louis.chaboche\}@onera.fr
}

\begin{abstract}
This paper presents a first validation of a novel methodology for identifying the parameters of a crystallographic elastoplastic constitutive law. This is accomplished by comparing simulation and experimental results at different length scales: the microstructure scale and the representative volume element scale. Experimentally, the microscopic strain fields and the microstrucural characteristics can be obtained only at the surface of the specimen. As a consequence, in finite element simulations only at the surface there is a oneto-one correspondence between the mesh and the experimental observed grain morphology. In this paper, the morphology of the subsurface grains is obtained by a simple extension in the thickness direction of the surface morphology. The aim of this study is then to verify whether the surface data contain sufficient information for the identification of the parameters of the constitutive law.

RÉSUMÉ. Cet article présente les premiers pas vers la validation d'une méthodologie. Cette dernière permet d'identifier les coefficients d'une loi de comportement elasto-plastique cristallographique par une comparaison directe des résultats numériques et expérimentaux obtenus à deux échelles : l'échelle de la microstructure et l'échelle macroscopique. Avec les moyens expérimentaux utilisés, les mesures de champs de déformation ainsi que la caractérisation de la microstructure ne peuvent être réalisées qu'à la surface de l'éprouvette. Le maillage par éléments finis ne peut donc prendre en compte que la microstructure de surface, qui est ici extrudée. L'objectif de l'étude présentée consiste donc à vérifier la pertinence de l'utilisation, dans les simulations numériques, d'une microstructure extrudée pour l'identification des coefficients de la loi de comportement.

KEYWORDS: EBSD, DIC, identification, crystallographic constitutive law, polycrystal, SEM.

MOTS-CLÉS : EBSD, corrélation d'images numériques, identification, loi de comportement elastoplastique cristallographique, polycristal, microscopie électronique à balayage.
\end{abstract}

DOI:10.3166/EJCM.18.333-351 @ 2009 Lavoisier, Paris

EJCM - 18/2009. Pictures and finite elements, pages 333 to 351 


\section{Introduction}

Striving to improve the mechanical behaviour of materials, aeronautical as well as automotive industries are interested in understanding the influence of microstructural parameters on the global mechanical properties. This influence can be analysed by using finite element (FE) calculations on virtual microstructures. By modifying microstructural parameters such as grain size and texture, the influence of each parameter can be evaluated independently, which is almost impossible to do by experimental investigations.

Simulations of microstructures have become more and more efficient and can now reproduce realistic crystallographic and morphological textures from statistical experimental data (St-Pierre et al., 2008; Saylor et al., 2004). By bringing these tools together, the challenge is now to improve the way to find the parameters of the constitutive elastoplastic law that reproduce not only the macroscopic behaviour, but also the intragranular one. Indeed, the correct description of the strain fields at the scale of the microstructure is a key point for a better understanding of damage initiation.

When the form of the constitutive law has been established, the next step is to affect numerical values to its parameters. These values can be identified by an inverse method in order to reproduce the experimental results (Claire et al., 2004; Grédiac et al., 2002; Meuwissen et al., 1998; Hoc et al., 2003). The development of digital image correlation (DIC) in association with Scanning Electron Microscope (SEM) images (Héripré et al., 2007; Soppa et al., 2001; Cornille, 2005; Kolednik et al., 2008) can provide strain field measurements at the intragranular scale. This field can be compared directly to the ones that are obtained numerically, for instance by FE simulations. This assumes that the FE mesh represents the real microstructure and not just a realistic one. Even though the EBSD technique offers the possibility to measure the crystallographic orientations (Euler angle triplets) of each grain of the surface microstructure, the 3D morphological texture cannot be accessed with this kind of technique. The creation of the mesh from the EBSD map can then only be either a 2D mesh or a 3D mesh obtained by an extension of the 2D surface.

The aim of this paper is then to validate the methodology proposed in (Héripré et al., 2007) with a special focus on the influence of the volumetric microstructure and of the boundary conditions. In a first part, the methodology that couples experimental investigations at the microscale with FE simulations is briefly presented. Then, numerical investigations are performed in order to evaluate the influence of the microstructure below the surface on the strain fields at the surface. As this subsurface microstructure cannot be characterised with EBSD analysis, the question is: do we need to have this information for the finite element simulations in order to be able to compare the numerical results with the experimental microscopic strain fields? In order to obtain some insight into this question, the surface strain fields of several different microstructures are compared quantitatively. Also, their averaged stress-strain curves are compared. These microstructures have all the same grain morphology and orientations at the upper surface, but below this surface their microstructures differ. 
For this study, a 3D simulated microstructure has been considered as a virtual experiment, and the methodology has been applied to this virtual experiment. With this choice, the methodology can be applied to a "reference" experiment for which every parameter is known: the $3 \mathrm{D}$ microstructure, the boundary conditions $(\mathrm{BC})$ as well as the parameters of the "reference" constitutive law. With this assumption, the validity of the methodology can be verified and its limits given.

\section{Methodology}

The methodology for the parameter identification of the crystallographic elastoplastic constitutive law consists of a simultaneous comparison at the macroscale and at the microscale between experimental data and results of FE calculations carried out on the real microstructure. The different steps of this methodology have been described and analysed in a previous paper (Héripré et al., 2007). Here is a brief summary in order to describe the context and the main objective of the present paper.

First, the microstructure of a preselected surface area $(\mathcal{A})$ is analysed by using the Electron Back-Scatterring Diffraction (EBSD) technique, providing a microstructure field which characterises the morphology of the grains as well as their crystallographic orientation (Figure 1(a)). A part of this 2D microstructure can then be meshed and an extension in the direction normal to the surface is performed (Figure 1(b)). Then a crystallographic elastoplastic constitutive law is used that takes into account the crystallographic orientation of each grain of the mesh.

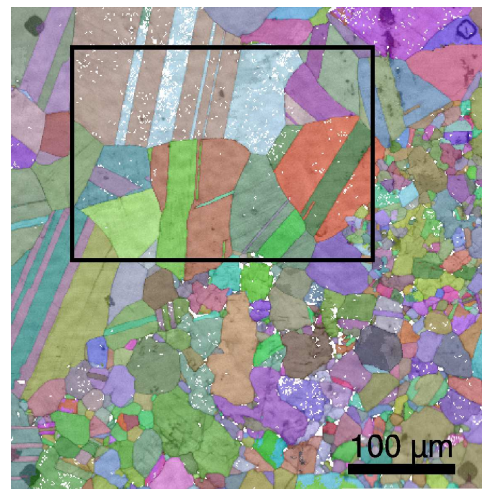

(a) EBSD map

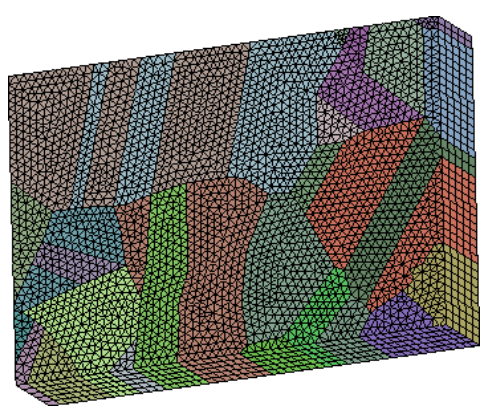

(b) The 3D FE mesh. The upper surface of this mesh corresponds to the black rectangle in (a)

Figure 1. Illustration of the first steps of the methodology applied to a Ti-48Al-2Cr$2 \mathrm{Nb}$ alloy, obtained by a powder metallurgy process

Secondly, a microgrid is deposited on the area $\mathcal{A}$ (Figure 2(a)). This microgrid is necessary to provide sufficient contrast at the intragranular scale for the DIC. Reference SEM images of the microgrid are taken before carrying out the mechanical test. 
Images of the area $\mathcal{A}$ are then taken at different times during the loading stage. Subsequent DIC applied to these images allows the determination of strain fields at the scale of the microstructure, as shown in Figure 2(b).

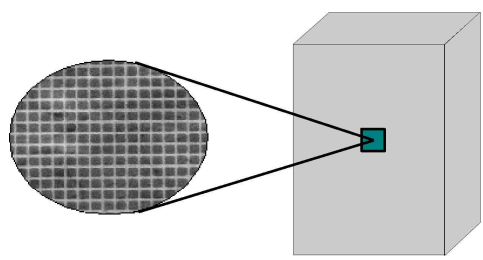

(a) Deposition of the microgrid

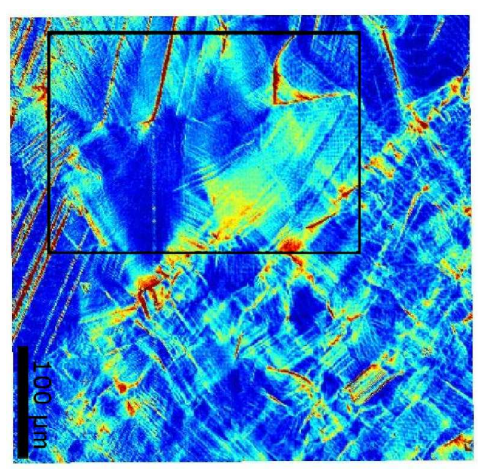

(b) Strain map. Grid step: $1 \mu \mathrm{m}$

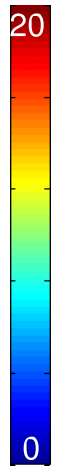

Figure 2. Illustration of the next steps of the methodology, applied to a Ti-48Al-2Cr$2 \mathrm{Nb}$ alloy, obtained by a powder metallurgy process

Finally, the finite element calculation can be carried out. The boundary conditions used are the experimental displacements, i.e. the in-plane displacements as measured by the DIC at each node of the mesh boundary. This choice seems to be necessary, in order to take into account the influence of the neighbouring grains, when an area smaller than the representative volume element is meshed. This will be discussed in the next sections. The numerical results can then be compared to the strain field at the scale of the grains as well as to the average strain and stress. The minimisation of the discrepancy between the numerical and experimental results leads to the identification of the parameters of the constitutive law.

In this case, the law is an elasto-visco-plastic crystallographic one with linear hardening (Cailletaud, 1988; Cailletaud et al., 2003) with only few parameters to identify. We take classically:

$$
\underline{\underline{\dot{\sigma}}}=\underline{\underline{\underline{\underline{K}}}}:\left(\underline{\underline{\dot{\varepsilon}}}-\underline{\underline{\varepsilon}}^{v}\right),
$$

where $\underline{\underline{\underline{K}}}$ is the tensor of elasticity, $\underline{\underline{\underline{\sigma}}}$ the stress rate, and $\underline{\underline{\underline{\varepsilon}}}$ and $\underline{\underline{\varepsilon}}^{v}$ the rates of total and viscoplastic strain, respectively. The activation of the slip system $s$, defined by slip plane normal $\underline{n}_{s}$ and the slip direction $\underline{m}_{s}$ is controlled by the Schmid law, where first activation of the slip system occurs if:

$$
\left|\tau_{s}\right|-\tau_{0 s} \geq 0,
$$

where $\tau_{s}=\underline{\underline{\sigma}}: \underline{\underline{R_{s}}}$ is the resolved shear stress on slip system $s$ and $\tau_{0 s}$ is its critical resolved shear stress. $\underline{\underline{R_{s}}}=\frac{1}{2}\left(\underline{n}_{s} \otimes \underline{m}_{s}+\underline{m}_{s} \otimes \underline{n}_{s}\right)$ is the orientation matrix of the 
system $s$. The viscoplastic strain increment due to the active slip systems $s$ is given by

$$
\underline{\underline{\varepsilon}}^{v}=\sum_{s} \dot{\gamma}_{s} \underline{\underline{R_{s}}}
$$

where $\dot{\gamma}_{s}$ is the shear rate on the slip system $s$, which follows a Norton law:

$$
\dot{\gamma}_{s}=\operatorname{sign}\left(\tau_{s}\right) \dot{v}_{s}
$$

with

$$
\dot{v}_{s}=\left\langle\frac{\left|\tau_{s}\right|-r_{s}}{K}\right\rangle^{n}
$$

and the Macauley bracket

$$
\langle a\rangle= \begin{cases}a & \text { if } a>0 \\ 0 & \text { if } a \leq 0\end{cases}
$$

and $\mathrm{K}$ and $\mathrm{n}$, material parameters. For each system $s$, the evolution of $r_{s}$ is linked to the cumulated shear by:

$$
r_{s}=\tau_{0 s}+h_{0} \sum_{k} h_{s k} v_{k}
$$

where $\underline{h}$ is the interaction matrix between the slip systems and $h_{0}$ the hardening parameter. For simplicity, we neglect the time dependence of the shear rate: with $n$ large $(\sim 20)$ and $K$ small $(\sim 1 \mathrm{MPa})$, this law becomes elastic-plastic in the time-independent limit.

However, this methodology is based on a comparison between the in-plane fields measured at the surface and those generated by $3 \mathrm{D}$ simulations. The microstructure below the surface obviously influences the fields at the surface, but the exact manner in which this occurs is not yet clear. In the next sections this key point is further investigated.

\section{Influence of the microstructure below the surface on the surface strain field analysis}

The first investigation carried out consists of FE calculations on different 3D microstructures having the same surface. These microstructures have been constructed by using Micro3D, a program that can create microstructures below a fixed surface (the one observed experimentally) by taking into account every statistical characteristic of the real microstructure, as given by EBSD analysis. Full details about the methodology can be found in (St-Pierre et al., 2008). 
Three microstructures with the same upper surface have been created (see Figure 3). For the set-up of the methodology, the grains are supposed to have a hexagonal close-packed (HCP) lattice, in which for simplicity only one family of dislocation slip systems can be activated: the $\beta$ prismatic system $(01 \overline{1} 0)[\overline{2} 110]$. With this assumption, only two parameters have to be optimised: the critical resolved shear stress $R_{0}=\tau_{0 \beta}$ (eq. 2) and the hardening parameter $H=h_{0} h_{11}$ (eq. 7). For the calculations presented in this section, the reference values of these parameters are $R_{0}=40$ $\mathrm{MPa}$ and $H=50 \mathrm{MPa}$.

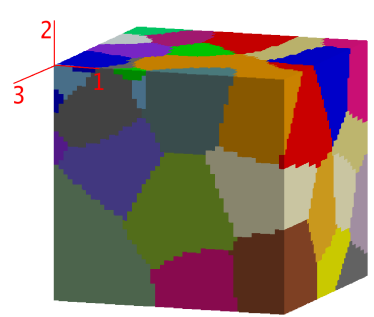

(a) Microstructure 1

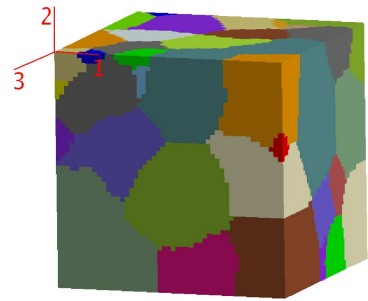

(b) Microstructure 2

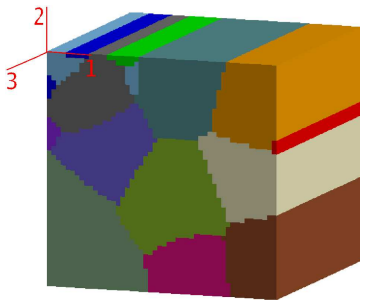

(c) Microstructure 3

Figure 3. Three simulated microstructures with the same surface and different reconstructed volumes

As shown by Figure 4, these three microstructures have quite the same orthotropic crystallographic texture with the strongest texture obtained for the extruded microstructure, which is a direct consequence of the extrusion that conserves and reinforces the surface texture.

The same loading conditions are applied to each mesh: homogeneous displacement is applied at the nodes of the top and bottom surfaces in direction 2 up to a maximum value of $0.125 \mu \mathrm{m}$, representing an average strain of $0.25 \%$. The other surfaces are left free. Two nodes are fixed in the direction normal to the surface (direction 3 ) and one node is fixed in direction 1 in order to prevent rigid body motions.

The results of the numerical calculations are presented in Figure 5.

The localisation of the highest strain is different from one calculation to another, as also observed by (Zeghadi et al., 2007). The higher strains are localised preferentially near grain boundaries, but they are strongly dependent on the microstructure below the surface. The distribution of strain intensity at each integration point of the surface elements has also been studied. This study is presented in Figure 6.

The strain distributions obtained on meshes 1 and 2 are quite similar but differ from the results coming from the extruded mesh. The latter have a stronger heterogeneity represented by a $50 \%$ higher standard deviation.

These results can lead to the conclusion that, if the aim of such a study is the optimisation of parameters by comparing numerical results with experimental strain fields at the grain scale, the entire 3D microstructure has to be simulated in order to be compared to the experimental results. However, the grain morphology is not the 


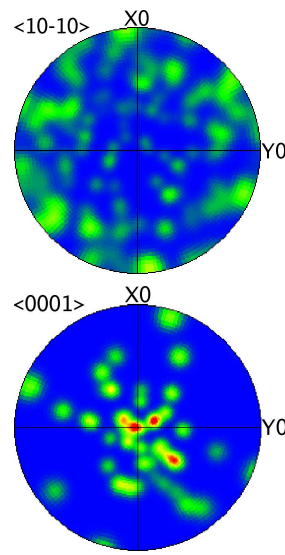

(a) Microstructure 1
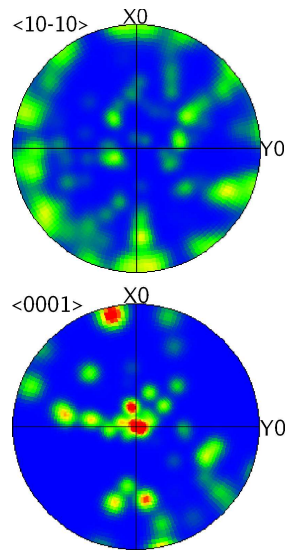

(b) Microstructure 2
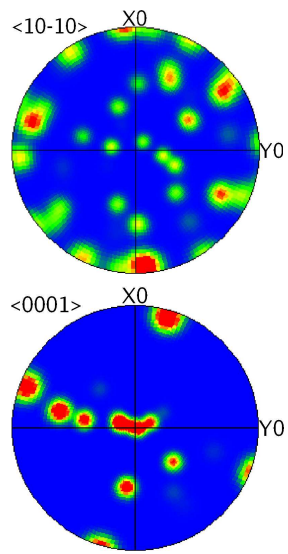

Densities (mud):

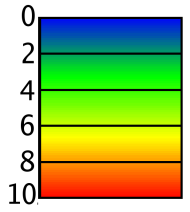

(c) Microstructure 3

Figure 4. Crystallographic texture of the three simulated microstructures of Figure 3. Crystal symmetry: 6/mmm - Stereographic projection for 2500 data points - Upper hemispheres

(a) Mesh 1:

Microstructure 1

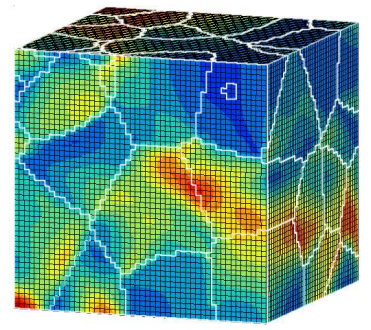

$\varepsilon_{22}-\min : 0.082-\max : 0.895$ (b) Mesh 2:

Microstructure 2

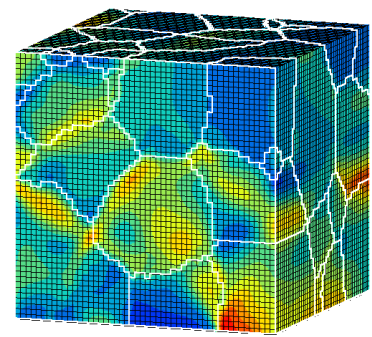

$\varepsilon_{22}-\min : 0.047-\max : 0.625$ (c) Mesh 3:

Microstructure 3

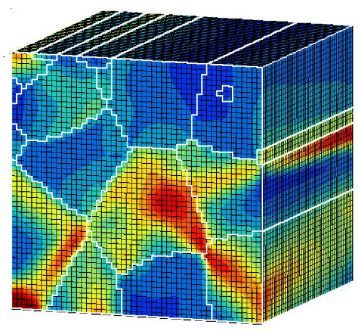

$\varepsilon_{22}-\min : 0.026-\max : 1.087$

$0 \%$

$0.7 \%$

Figure 5. Influence of the microstructure below the surface on surface strain fields Test on a microstructure with HCP lattice - 397953 dof

only factor having a strong influence on the surface strain field. Also, the manner in which the boundary conditions are applied plays a crucial role. The key question is now to determine how the homogeneous boundary conditions applied to the mesh influence the strain field in order to obtain the proper parameters of the constitutive law by comparison of the numerical results with the experimental strain fields? This is the aim of the next section. 


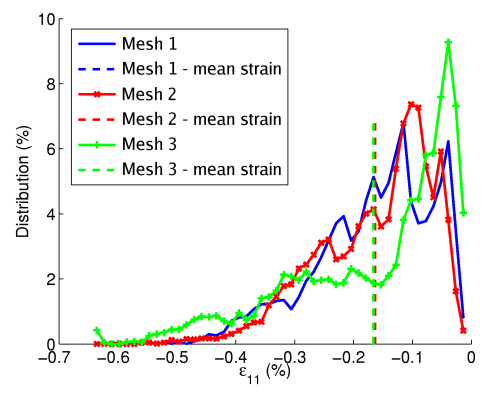

\begin{tabular}{|c|c|c|}
\hline & mean strain & st. deviation \\
\hline Mesh 1 & $-0.17 \%$ & $0.10 \%$ \\
Mesh 2 & $-0.16 \%$ & $0.10 \%$ \\
Mesh 3 & $-0.17 \%$ & $0.14 \%$ \\
\hline
\end{tabular}

(a) Transversal strain $\varepsilon_{11}$

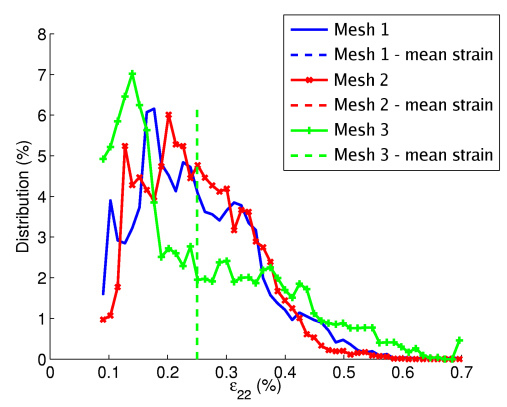

\begin{tabular}{|c|c|c|}
\hline & mean strain & st. deviation \\
\hline Mesh 1 & $0.25 \%$ & $0.10 \%$ \\
Mesh 2 & $0.25 \%$ & $0.09 \%$ \\
Mesh 3 & $0.25 \%$ & $0.14 \%$ \\
\hline
\end{tabular}

(b) Longitudinal strain $\varepsilon_{22}$

Figure 6. Distribution of surface strain for the three meshes of Figure 3

\section{Influence of the boundary conditions on an extruded microstructure}

In the methodology presented in section 2, experimental displacements are applied at each node of the edges of the extruded surface. That means that this takes into account the interaction of the neighbouring grains of the surface, but there is probably also some influence of the subsurface grains. The aim of the further study is then to evaluate the influence of the boundary conditions on the surface strain field. In other words, this study is intending to evaluate whether the knowledge of the real boundary conditions, as measured by DIC, can somehow compensate the lack of knowledge of the subsurface microstructure. If the latter is true, it means that numerical simulations carried out on an extrusion of the surface mesh can be compared to measurements of strain fields at the surface of the specimen.

In order to answer these questions, the 3D simulated microstructures have been considered now as "reference microstructures". This gives the possibility to have a well-known test since the boundary conditions are completely known, as well as the full 3D microstructure and even the parameters of the constitutive law, which are the parameters to be identified in the general case. In this section, the results of the virtual test will be referred as "reference" results.

For these virtual 3D microstructures, smaller zones of the surface have been selected as illustrated in Figure 7, exactly in the same manner as in the methodology presented previously (see Figure 1). 

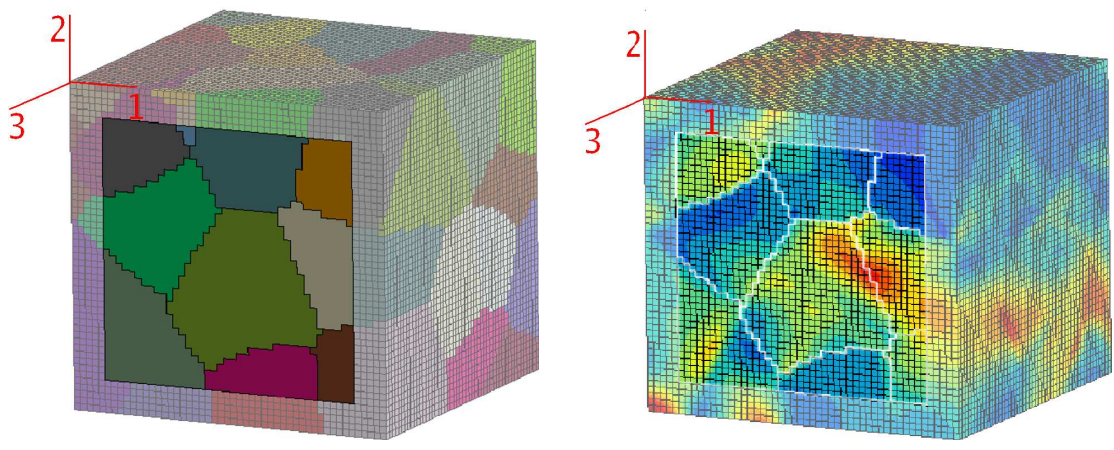

Figure 7. Selection of a surface area for the study of the influence of the boundary conditions

Then, these areas can be extruded and two types of boundary conditions have been studied: homogeneous and "reference" ones, that come from the virtual test. Homogeneous means that the same displacement has been applied to each node of the top and bottom boundaries (i.e. with surfaces normal to the direction 2), and the other boundaries are left free. Experimental boundary conditions means that the "reference" displacements, measured at the surface edges of the microstructure, are applied at each node of the mesh boundaries while the surfaces normal to the 3 -axis are left free. In this case, the nodes of the surfaces normal to axes 1 and 2 have the same displacement if they have same coordinates $\mathrm{x} 1$ and $\mathrm{x} 2$ but different coordinate $\mathrm{x} 3$. This corresponds to an "extension" of the in-plane boundary conditions. The $\varepsilon_{22}$ strain components resulting from these numerical calculations are presented in Figure 8.

Figures 8 (a) and 8 (b) show the strong influence of the boundary conditions on the surface strain fields for microstructure 1 . Figures $8(\mathrm{c})$ and $8(\mathrm{~d})$ confirm, by quantifying the discrepancy between the 3D reference simulation and the two tested configurations, that experimental boundary conditions decrease this discrepancy. These errors on local strain components have been calculated by the normalised discrepancy between the results of surface strain fields on mesh 1 and mesh 2:

$$
\Delta_{i j}^{k}=\frac{\left|\varepsilon_{i j}^{2, k}-\varepsilon_{i j}^{1, k}\right|}{\left|\varepsilon_{i j}^{1, k}\right|}
$$

with $k$ denoting each integration point of the mesh, and $\varepsilon_{i j}^{n, k}$ components $i j$ of the strain tensor at integration point $k$ of the mesh $n$.

The results on the local strain in direction 2, which is the tensile direction, indicate that $29 \%$ of the local $\varepsilon_{22}$ have an error of less than $20 \%$ by applying homogeneous boundary conditions. As for the experimental boundary conditions, the number of integration points showing such an error or a lower one is equal to $68 \%$. 


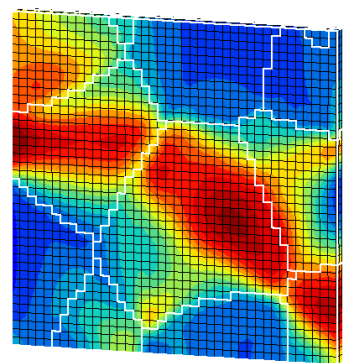

$\varepsilon_{22}-\min : 0.036-\max : 0.804$

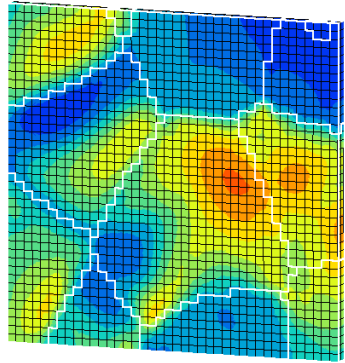

$\varepsilon_{22}-\min : 0.163-\max : 0.233$

$0 \%$

(a) $\varepsilon_{22}$ (vertical strain) for homogeneous displacements

$$
0.7 \%
$$

(b) $\varepsilon_{22}$ (vertical strain) for experimental displacements

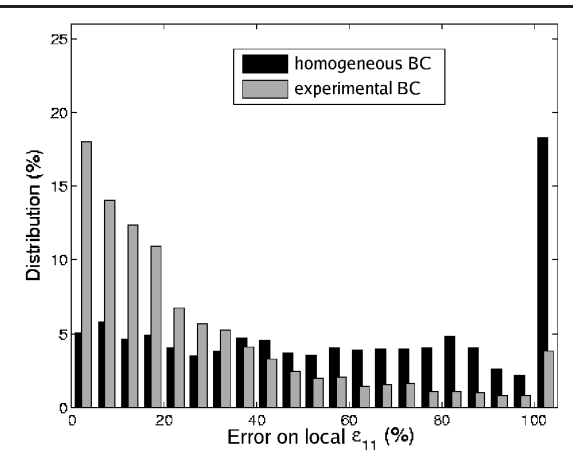

(c) Relative error w.r.t. reference $\varepsilon_{11}$

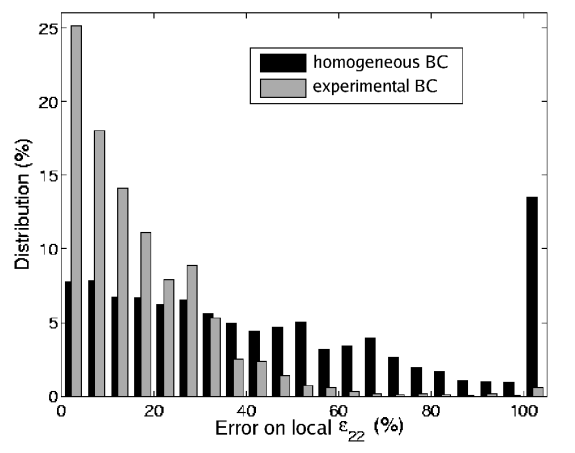

(c) Relative error

w.r.t. reference $\varepsilon_{22}$

Figure 8. Influence of the boundary conditions on the strain fields at the surface

These results have been obtained for a very thin mesh (with one element in the thickness). However, the influence of the thickness on the strain field has also been studied. Three cases are shown in Figure 9 with a thickness defined by (a) only one element, (b) 15 elements and (c) 15 elements with symmetry conditions for the lower surface, which is somewhat equivalent to 30 elements.

The surface $\varepsilon_{22}$ strain fields show a stronger dependence on the thickness when homogeneous BC are applied (Figure 9(2)), as also observed with the histograms representing the distributions of local $\varepsilon_{11}$ and $\varepsilon_{22}$ components of strain fields (see figures 10 and 11). 


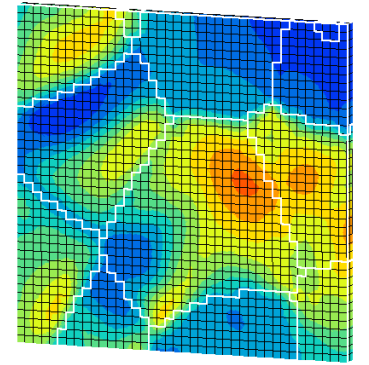

$\varepsilon_{22}-\min : 0.163-\max : 0.233$

(a1)

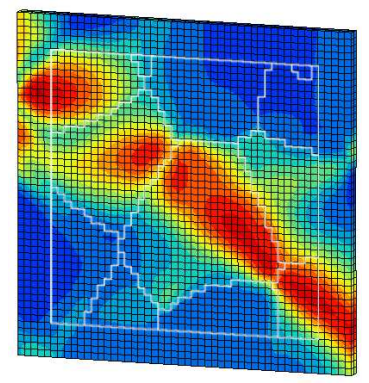

$\varepsilon_{22}-\min : 0.055-\max : 0.658$

(a2)

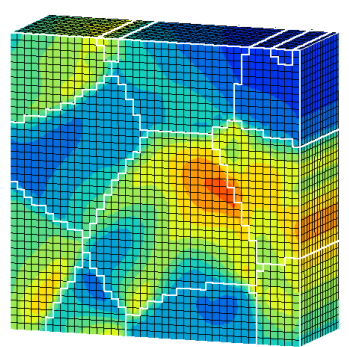

$\varepsilon_{22}$ - min:0.057 - max:0.540

(b1)

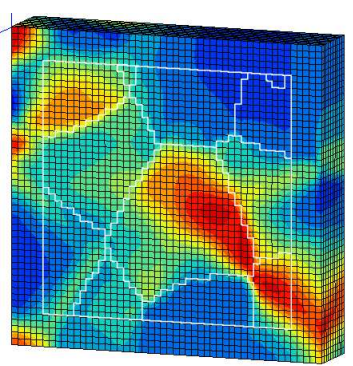

$\varepsilon_{22}$ - min:0.015 - max:1.020

(b2)

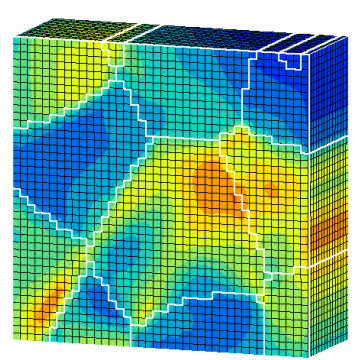

$\varepsilon_{22}-\min : 0.047-\max : 0.523$

(c1)

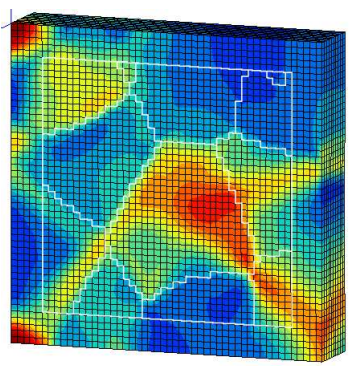

$\varepsilon_{22}-\min : 0.036-\max : 0.804$

(c2)

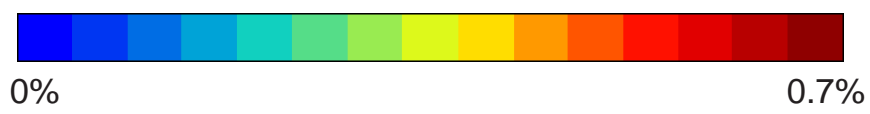

Figure 9. Influence of mesh thickness on the surface strain field (1) for experimental boundary conditions and (2) for homogeneous boundary conditions

As for the experimental BC, the errors for the third case are greater than those of the second case. More integration points have an error greater than $30 \%$ for the third case than for the second one (Figure 10). This means that there exists an optimum size of the thickness when applying experimental boundary conditions, which seems to be of the order of the grain size.

When applying homogeneous boundary conditions, the errors decrease when increasing the thickness (figure 11).

Then, the application of experimental boundary conditions seems to make possible the comparison with experimental results, because the simulated strain field reproduces the real strain heterogeneity quite well. The same validation has been performed on the second microstructure (Figure 3(b)). The results are presented in figure 12. 

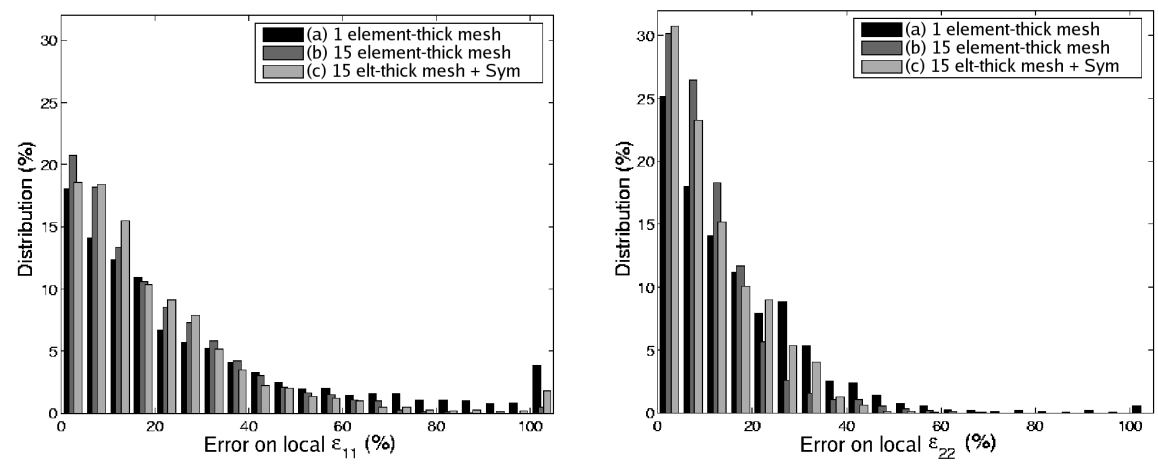

Figure 10. Influence of the mesh thickness on the relative error of the local strain field - Experimental boundary conditions
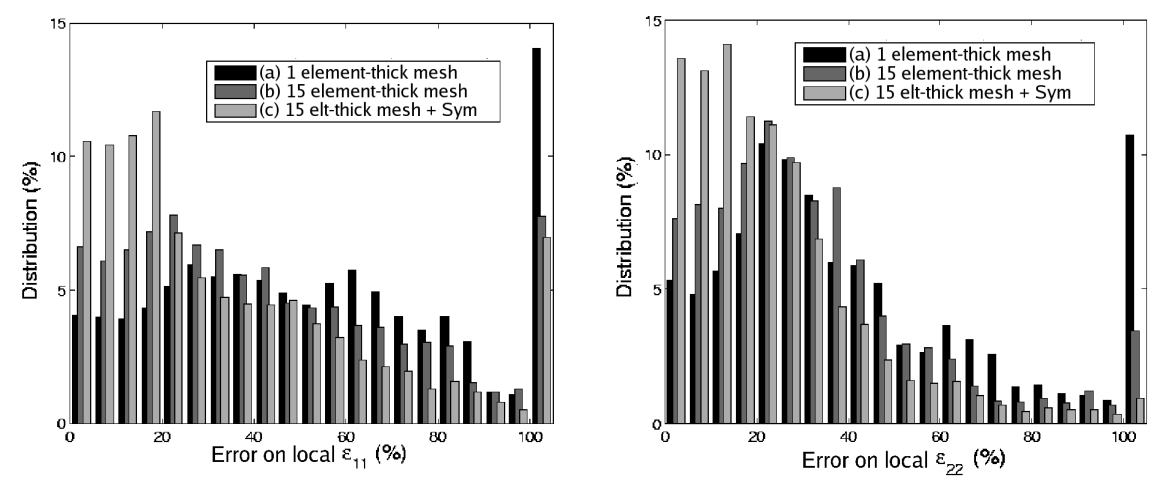

Figure 11. Influence of the mesh thickness on the relative error of the local strain field - Homogeneous boundary conditions

The thickness of the mesh is equal to 15 elements with free lower and upper surfaces let free which gives the best results for the microstructure 1. In this case, $80 \%$ of the $\varepsilon_{22}$ at the integration points have a difference with the experimental results of less than $20 \%$, which is better than the errors for the analysis on microstructure 3. Also, almost all of $\varepsilon_{11}$ at the integration points have an error less than $20 \%$.

As has been demonstrated in this section, applying experimental BC on an extruded mesh (at least in these two cases) can reproduce the experimental strain field at the scale of the microstructure. This has been verified by applying the methodology on a virtual reference test i.e. a tensile test on a simulated microstructure. The strain field at the surface is similar to the reference one, but is the error between the two results the minimum possible? In other words, would it be possible, by changing the 
parameters of the constitutive law, to minimise this error even more (i.e. with an incorrect constitutive law)? If this is not the case, this means that extrusion of the surface would be (probably) sufficient for the identification of parameters of the constitutive law. The next section will present this study.
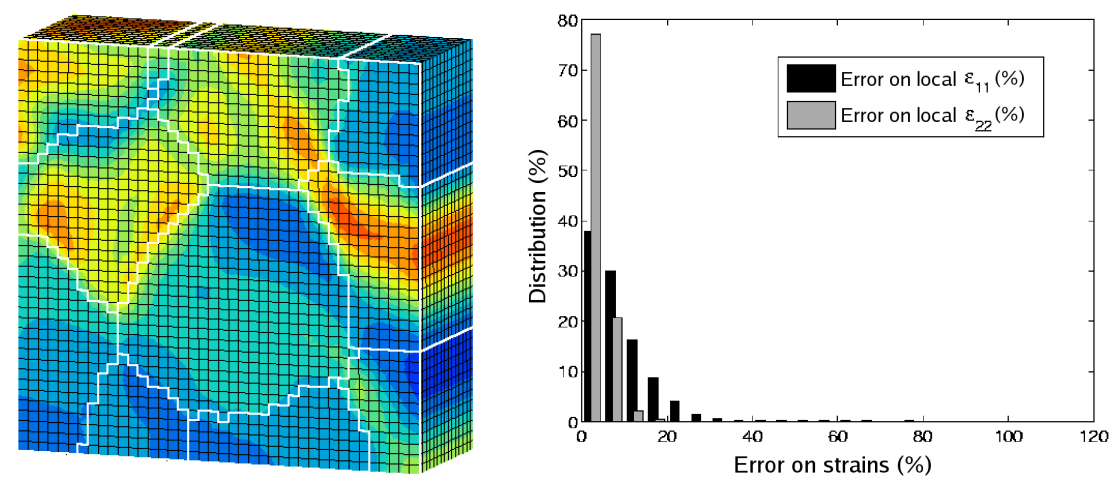

$\varepsilon_{22}-\min : 0.074-\max : 0.568$

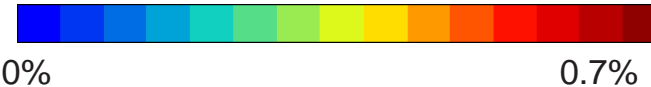

Figure 12. Validity of experimental boundary conditions applied to the second $3 D$ microstructure

\section{Optimisation of the parameters with the virtual test}

In order to answer this last question, the microstructure 1 is considered as the reference microstructure on which a virtual test is carried out. The cost function to minimise is classically defined as (Gürdal et al., 1992):

$$
\mathcal{F}^{k}=\frac{1}{2}\left(\underline{f}\left(\underline{x}^{k}\right)-\underline{y}\right)^{T} \underline{\underline{W}}\left(\underline{f}\left(\underline{x}^{k}\right)-\underline{y}\right),
$$

where

$-\underline{x}^{k}$ represents vector of parameters to minimise. In this case, the vector contains two elements with the critical resolved shear stress $R_{0}$ and the hardening parameter $H$ of prismatic system (cf. fig. 13).

- $\underline{f}\left(\underline{x}^{k}\right)$ is the vector of the numerical results,

$-\underline{y}$ is the vector of the experimental results and

$-\underline{\underline{W}}$ is the positive matrix containing weights associated to each comparison. 


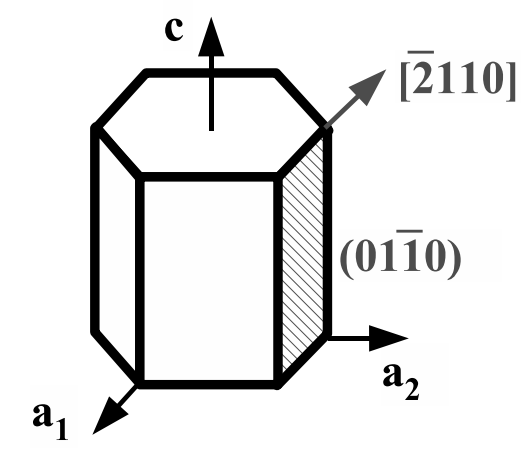

Figure 13. One of the three prismatic slip systems of a HCP lattice

The cost function is then defined by taking into account the macroscopic response (average stress and strain on the aggregate) as well as the microscopic response i.e. the displacements of each node of the surface or the strains at each integration point located near the surface. Other choices can be considered as well, such as the mean strain inside grains, etc. The analysis of the definition of the cost function is not the topic of this paper, but has to be carried out in the future for a complete confirmation of the methodology. In this case, displacements of each node of the surface have been used. The dimension of $f\left(\underline{x}^{k}\right)$ and $y$ is then $\left(2 \times N_{n}+3 \times N_{M}\right)$, with $N_{n}$ the number of surface nodes and $N_{M}$ a number of points discretising the time evolution of the macroscopic stres-strain curve. These vectors are then composed of the displacements in the horizontal direction 1 , and in the vertical direction 2, and the mean $\sigma_{11}$ and $\sigma_{22}$ stresses and $\varepsilon_{22}$ strain at each time step. $\underline{W}$ is composed of the inverse of squared mean values ( $u_{1}$ and $u_{2}$ mean value on all nodes and $\varepsilon_{22}, \sigma_{11}$ and $\sigma_{22}$ mean values)

The cost function can then be written as:

$$
\mathcal{F}^{k}=\frac{1}{2}\left(\frac{E_{M 1}}{A_{M 1}^{\text {exp }}}+\frac{E_{M 2}}{A_{M 2}^{\text {exp }}}+\frac{E_{M 3}}{A_{M 3}^{\text {exp }}}+\frac{E_{L 1}}{A_{L 1}^{\text {exp }}}+\frac{E_{L 2}}{A_{L 2}^{\text {exp }}}\right)
$$

where $E_{M 1}$ and $E_{M 3}$ represent the error on the mean strain and stress in the tensile direction, and $E_{M 2}$ the error on the mean stress in the transverse direction:

$$
\begin{aligned}
& -E_{M 1}=\sum_{i=1}^{N_{M}}\left(\varepsilon_{22}\left(t_{i}\right)-\varepsilon_{22}^{e x p}\left(t_{i}\right)\right)^{2} \\
& -E_{M 2}=\sum_{i=1}^{N_{M}}\left(\sigma_{11}\left(t_{i}\right)-\sigma_{11}^{e x p}\left(t_{i}\right)\right)^{2} \\
& -E_{M 3}=\sum_{i=1}^{N_{M}}\left(\sigma_{22}\left(t_{i}\right)-\sigma_{22}^{e x p}\left(t_{i}\right)\right)^{2}
\end{aligned}
$$

These values, representing the macroscopic behaviour, have to be normalised. The normalisation is performed by dividing $E_{M 1}, E_{M 2}$ and $E_{M 3}$ by $A_{M 1}^{\exp }, A_{M 2}^{\exp }$ and $A_{M 3}^{\exp }$ respectively. 


$$
\begin{aligned}
& -A_{M 1}^{\text {exp }}=\frac{1}{N_{M}} \sum_{i=1}^{N_{M}}\left(\varepsilon_{22}^{e x p}\left(t_{i}\right)\right)^{2} \\
& -A_{M 2}^{e x p}=\frac{1}{N_{M}} \sum_{i=1}^{N_{M}}\left(\sigma_{11}^{e x p}\left(t_{i}\right)\right)^{2} \\
& -A_{M 3}^{e x p}=\frac{1}{N_{M}} \sum_{i=1}^{N_{M}}\left(\sigma_{22}^{e x p}\left(t_{i}\right)\right)^{2}
\end{aligned}
$$

In the same manner, the local error is taken into account with the difference between experimental and numerical displacements at each node of the surface of the mesh. The local errors $E_{L 1}$ and $E_{L 2}$ in the displacements in directions 1 and 2 respectively are then introduced and normalised by $A_{L 1}^{\exp }$ and $A_{L 2}^{\exp }$ :

$$
\begin{aligned}
& -E_{L 1}=\sum_{i=1}^{N_{n}}\left(u_{1}\left(x_{i}, y_{i}\right)-u_{1}^{e x p}\left(x_{i}, y_{i}\right)\right)^{2} \\
& -E_{L 2}=\sum_{i=1}^{N_{n}}\left(u_{2}\left(x_{i}, y_{i}\right)-u_{2}^{e x p}\left(x_{i}, y_{i}\right)\right)^{2} \\
& -A_{L 1}^{\exp }=\frac{1}{N_{n}} \sum_{i=1}^{N_{n}}\left(u_{1}^{\exp }\left(x_{i}, y_{i}\right)\right)^{2} \\
& -A_{L 2}^{\exp }=\frac{1}{N_{n}} \sum_{i=1}^{N_{n}}\left(u_{2}^{\exp }\left(x_{i}, y_{i}\right)\right)^{2}
\end{aligned}
$$

With this definition of the cost function, the macroscopic strain-stress curve is represented properly. The macroscopic transverse stress, which can cause some problems when the evolution of boundary conditions is not realistic, as mentioned in section 6 , is taken into account in order to be correctly represented as well. Finally, the local information is considered by including, in the cost function, the error on experimental displacements, as measured by the DIC technique, and numerical displacements at each node of the surface of the mesh.

The results of the uniaxial tension test carried out on microstructure 1 are considered as the reference results, as previously. Then, the in-plane components of the displacement field at the surface obtained with the calculation on the 15-elements extrusion of the selected area are compared with those of the 3D reference calculations. For simplicity, only one family of slip system is taken into account, so only two parameters have to be identified. The evolution of the cost function with the variation of these parameters can then be visualised, as presented in figure 14.

This figure shows that a minimum is clearly defined for the parameter $R_{0}$, i.e. the critical resolved shear stress, but this is not the case for the hardening parameter $H$. The variation of the cost function as each of these parameters evolves towards their minima $R_{0}=40 \mathrm{MPa}$ and $H=50 \mathrm{MPa}$ (the reference values of the parameters that have been used for virtual test) is presented in figures 15(a) and 15(b) respectively.

These figures underline the well-defined minimum for $R_{0}$ and the absence of a clear minimum for $H$. This can be explained by the value of average strain: there is not much plasticity inside the microstructure (the average total strain of $0.25 \%$ is only 
just beyond the yield point at $0.20 \%$ ), which means that the parameter $H$ has only a weak influence on the results. So, to improve the accuracy on $H$, the tests have to be carried further into the plastic domain. On the other hand, $R_{0}$, describing the onset of plasticity, is well defined.

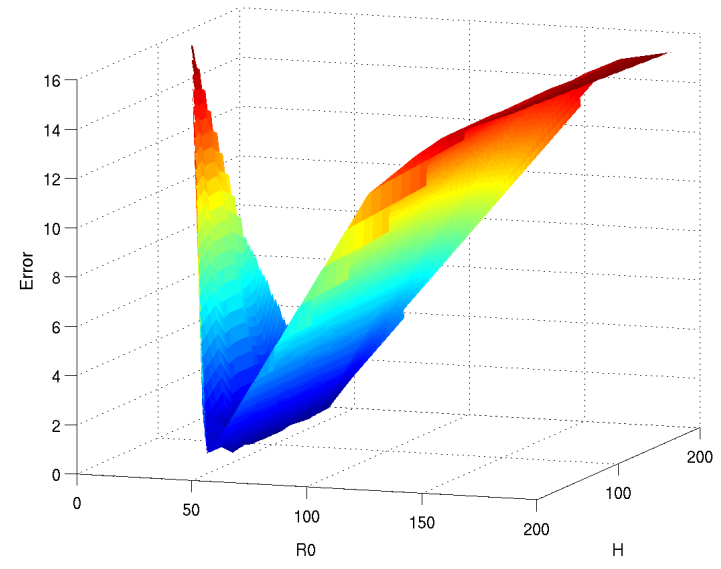

Figure 14. Variation of the cost function for the extruded mesh

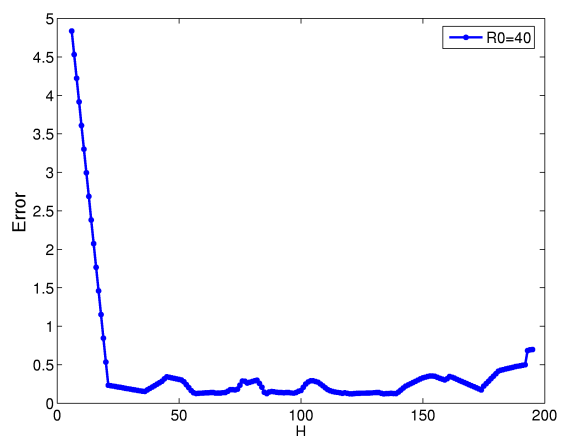

(a) Variation of the error for $R_{0}=40 \mathrm{MPa}$

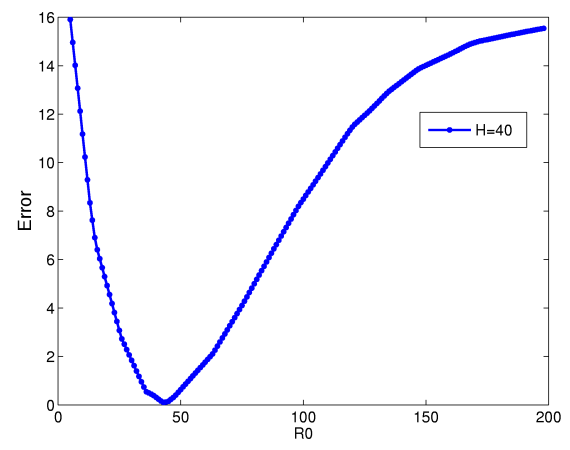

(b) Variation of the error for $H=50 \mathrm{MPa}$

Figure 15. Variation of the error w.r.t. values of the parameters $R_{0}$ et $H$ 

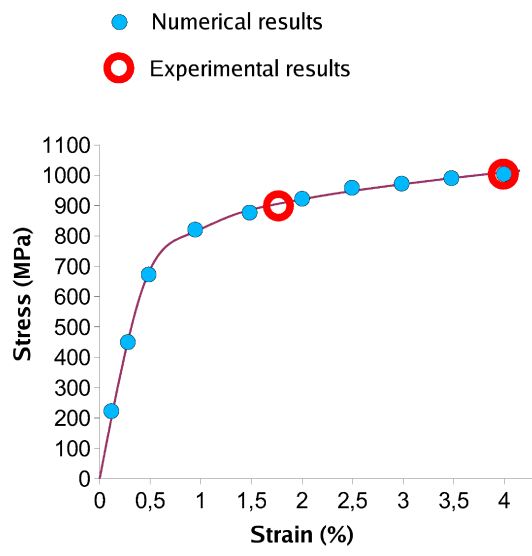

Figure 16. Illustration of the time discretisation of the measurement

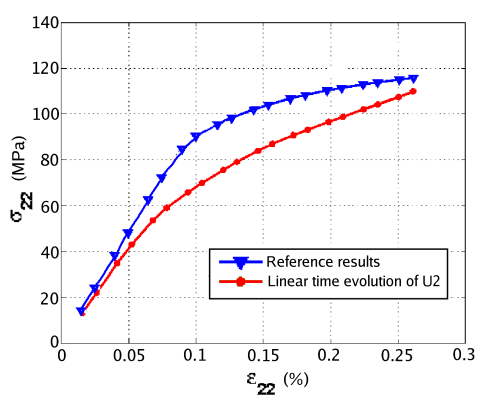

(a) Macroscopic stress-strain curve in the loading direction

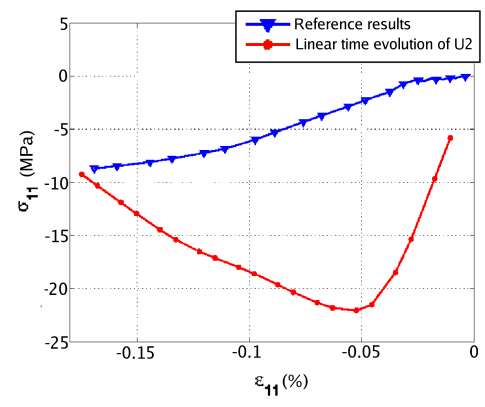

(b) Transversal stress $\sigma_{11}$

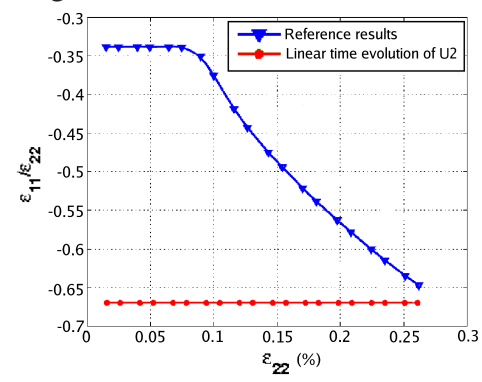

(c) Strain ratio $\varepsilon_{11} / \varepsilon_{22}$

Figure 17. Influence of a linear time evolution of displacements $u_{1}$ on macroscopic results 


\section{Conclusion and discussion}

This paper investigates the validity of a comparison between experimentally determined surface strain fields at the scale of the microstructure and the numerical calculation performed on the surface microstructure without identifying the real 3D microstructure because of the difficulties to obtain it without using non-destructive methods as for instance synchrotron facilities. In the presented methodology EBSD analysis is used. This means that only the morphology and grain orientation of microstructure of the surface is known. The aim of this paper was then to analyse the discrepancy between FE calculations performed on an extruded surface with FE calculations on a virtual 3D microstructure, presenting exactly the same surface.

The results have underlined that by applying the experimental in-plane boundary conditions, i.e. the displacements measured by DIC at each node of the mesh boundaries of the upper surface, the numerical strain fields of the surface are almost equivalent to the experimental results (or, in this study, results on 3D virtual microstructure). The difference that subsists seems to be the minimum of the cost function (at least for the critical resolved shear stress in this case).

Other analyses still have to be carried out for a complete validation of this methodology. One of the further analyses is the description of the time evolution of the boundary conditions. For instance, the reference results here are purely numerical, and in a certain sense, perfect. When the real experiment is used as reference, other sources of error have to be taken into account, such as the accuracy of the DIC method (quite important in the case of SEM images), the appropriateness of the constitutive law that has been chosen, or the size of the representative volume element, that has to be specified, etc. The resolution of the strain field obtained by DIC carried out on SEM images is not high enough to be able to catch elastic strains. Therefore, the first measurement point is obtained already well beyond the macrosopic yield limit, as illustrated by figure 16, whereas the results given by the virtual test can give "measurements" even in the elastic part of the macroscopic curve. If the goal is the identification of critical resolved shear stresses, the onset of plasticity has to be described precisely in order to identify realistic parameters. However, if it is assumed that the variation of displacements at each node is linear, this introduces transversal macroscopic stresses as illustrated by figure 17(b), because the first experimental point lies in the plastic domain, for which $\frac{\varepsilon_{11}}{\varepsilon_{22}}$ is equal to 0.5 instead of $\nu$ in the elastic domain (figure 17(c)).

Some assumptions should then be adopted to ensure a correct description of the transition between elastic and plastic part of the mechanical response. Moreover, this study has been carried out on a very small part of the microstructure. In this case, the influence zone of the boundary conditions are probably of the same order as the entire mesh. Though, the next studies would be devoted to the characterization of the largest size of the microstructure that can be used for the parameter optimisation. 


\section{Acknowledgements}

The authors are greatful to M. Thomas and J.-L. Raviart from the ONERA DMMP department for providing the TiAl samples and to D. Caldemaison from LMS for his help during in-situ experiments.

\section{References}

Cailletaud G., "Une approche micromécanique du comportement des polycristaux", Rev. Phys. App., vol. 23, p. 353-363, 1988.

Cailletaud G., Forest S., Jeulin D., Feyel F., Galliet I., Mounoury V., Quilici S., " Some elements of microstructural mechanics”, Comp. Mat. Sc., vol. 27, n³, p. 351-374, 2003.

Claire D., Hild F., Roux F., “ A finite element formulation to identify damage fields: the equilibrium gap method”, Int. J. Numer. Meth. Engng., vol. 61, p. 189-208, 2004.

Cornille N., Accurate 3D Shape and Displacement Measurement using a Scanning Electron Microscope, Phd thesis, University of South Carolina, INSA Toulouse, 2005.

Grédiac M., Toussaint E., Pierron F., " Special virtual fields for the direct determination of material parameters with the virtual fields method. 2-Application to in-plane properties", Int. J. Sol. and Struct., vol. 39, p. 2707-2730, 2002.

Gürdal Z., Haftka R., Elements of Structural Optimization, Kluwer Academic Publishers, 1992.

Héripré E., Dexet M., Crépin J., Gélébart L., Roos A., Bornert M., Caldemaison D., " Coupling between Experimental Measurements and Polycrystal Finite Element Calculations for micromechanical study of metallic materials", Int. J. Plast., vol. 23, p. 1512-1539, 2007.

Hoc T., Crépin J., Gélébart L., Zaoui A., “ A procedure for identifying the plastic behavior of single crystals from the local response of polycrystals", Acta Mater., vol. 51, p. 5477-5488, 2003.

Kolednik ., Unterweger K., " The ductility of metal matrix composites - Relation to local deformation behavior and damage evolution", Engng. Fract. Mech., vol. 75, p. 3663-3676, 2008.

Meuwissen M., Oomens C., Baaijens F., Petterson R., Janssen J., “ Determination of elastoplastic properties of aluminium using a mixed numerical-experimental method", J. Mater. Proc. Tech., vol. 75, p. 204-211, 1998.

Saylor D., Fridy J., El-Dasher B., Jung K., Rollett A., “ Statistically Representative ThreeDimensional Microstructures Based on Orthogonal Observation Sections", Met. Mat. Trans. A, vol. 35A, p. 1969-1979, 2004.

Soppa E., Doumalin P., Binkele P., Wiesendanger T., Bornert M., Schmauder S., “Experimental and numerical characterisation of in-plane deformation in two-phase materials", Comp. Mater. Sc., vol. 21, p. 261-275, 2001.

St-Pierre L., Héripré E., Dexet M., Crépin J., Bertolino G., Bilger N., “ 3D simulations of microstructure and comparison with experimental microstructure coming from O.I.M analysis", International Journal of Plasticity, vol. 24, p. 1516-1532, 2008.

Zeghadi A., Forest S., Gourgues A., Bouaziz O., “ Ensemble averaging stress-strain fields in polycrystalline aggregates with a constrained surface microstructure - part 2 : crystal plasticity", Phil. Mag., vol. 87, n 8-9, p. 1425-1446, 2007. 
